\title{
SIMULATOR PERFORMANCE VS. NEUROPHYSIOLOGIC MONITORING: WHICH IS MORE RELEVANT TO ASSESS DRIVING IMPAIRMENT?
}

\author{
Henry J. Moller, Leonid Kayumov, Eric L. Bulmash, \\ Colin M. Shapiro, Sidney H. Kennedy \\ Sleep Research Unit and Human Performance Laboratory \\ Toronto Western Hospital \\ Department of Psychiatry \\ University Health Network \\ University of Toronto \\ Toronto, Canada \\ E-mail: hmoller@uhnres.utoronto.ca
}

Summary: Previously, we reported on circadian variation in driving simulator performance and neurophysiologic evidence of sleep intrusion into consciousness in a pilot study of healthy individuals. We have since expanded this "normative" sample and run a prospective comparison study with a sample of clinical patients reporting excessive daytime sleepiness (EDS) as a chief complaint. Thirty healthy adults (mean age of $31.3 \pm 11.5)$ and 27 EDS patients $(47.0 \pm 13.7)$ with valid driver's licenses were included. Subjects performed four intentionally soporific 30-minute driving simulations at two-hour intervals while undergoing continuous EEG monitoring for microsleep (MS) episodes. Measured variables included: subjective ratings of sleepiness and alertness prior to each drive, lane position accuracy, mean speed, speed deviation, mean reaction time (RT) to "virtual" wind gusts as well as off-road events, i.e., "crashes." In comparing normative individuals and EDS patients, significant between-group differences were found between subjective ratings, RT, crashes and MS. Both groups showed a significant a tendency towards RT slowing during afternoon drives, with this circadian effect appearing most pronounced for EDS patients. Significant between-group differences were also found on subjective ratings of sleepiness and alertness, although diurnal fluctuation of subjective sleepiness ratings was significant only for the EDS group. Objective EEG MS monitoring demonstrated escalating sleep intrusion with repeated drives in both groups, but particularly for the EDS group. Total crash rates were three times higher in EDS patients, with an increasing trend towards crash-proneness in the late afternoon. In summary, we found significantly impaired performance on some, though not all, driving parameters for EDS patients. While increased crash rate may be the most dramatic of these, slowing of RT was the most statistically robust. EEG monitoring was able to document increased propensity towards MS episodes in patients with EDS, which we suggest is causative in creating this impairment. It remains unclear whether a neurophysiologic or simulator approach captures impairment due to sleepiness with greater sensitivity and specificity. A hybrid approach combining data from both sources may be optimal, and also could be integrated in commercial vehicle use. We suggest that the need for a more accurate hospitalbased screening tool for assessment of driving impairment due to sleep disorders remains an important issue for physicians and legislators dealing with driving competency. 


\section{INTRODUCTION}

The risk of driving impairment due to sleepiness and fatigue is becoming increasingly appreciated by clinicians and the general public (MacLean, Davies \& Thiele, 2003). It may be optimistic to claim that research and legislation on this important issue is at the same stage as drinking and driving public policy was some 25 years ago (Room, Babor \& Rehm, 2005). Certainly there is a greater appreciation in terms of public awareness, but without a clinically and medicolegally accurate and ecologically valid measurement tool, it will remain challenging to make practical progress.

Impairment due to excessive daytime sleepiness, like impairment due to alcohol may fluctuate significantly (Arnedt et al., 2000). However, unlike exogenous substances, impairment due to sleep pathologies frequently represent intrinsic, medically-based neuropsychiatric pathologies, which fluctuate further with imposed sleep-restriction, concomitant use of medications, time of day and level of external/environmental stimulation (Moller, Shapiro \& Kayumov, 2004; Moller, Kayumov \& Shapiro, 2003). Common auxiliary symptomatologies of excessive sleepiness disturbances include depressed and/or mood, decreased short-term memory, and detriments in psychomotor or executive function (Alchanatis et al., 2005). Many of these symptoms may resolve with appropriate medical treatment.

In many North American jurisdictions, including Ontario, physicians are held medically responsible for reporting to the Ministry of Transportation any drivers that may be impaired due to a medical condition, including neuropsychiatric and sleep disorders (Determining Medical Fitness to Drive, 2000). Yet, no clearly established clinical protocols exist to guide clinicians' decision making. Current standard daytime tests to assess disorders of excessive sleepiness include the Mean Sleep Latency Test (MSLT) and Maintenance of Wakefulness Test (MWT) (Mitler, Carskaddon \& Hirschkowitz, 2000). We have previously suggested that these are thought to have low sensitivity and are not necessarily ecologically valid to the task of driving performance (Moller, Kayumov \& Shapiro, 2003). Furthermore, it is difficult to envision transfer of these polysomnographic (PSG) tests to an in-vehicle application for use in the transport industry.

To date, no study has tested the reproducibility of EEG changes that occur during driver fatigue. It should be noted that sleepiness/fatigue during driving is not a continuous process but consists of successive episodes of "microsleeps" where the subject's state of consciousness may fluctuate in and out of a sleep state (Lal \& Craig, 2005). We have developed a pilot in-hospital screening protocol specific to detecting driving impairment due to excessive sleepiness. This involved a procedure of continuous neurophysiologic/EEG monitoring during a series of four 30-minute simulator drives. This multi-drive protocol was developed to acknowledge the potential for circadian variation in sleepiness throughout the day, as we had reported previously at this meeting. Unlike our previous smaller sample examing circadian performance in normative subjects only (Moller, Kayumov \& Shapiro, 2003), in our current experimental design, we have sought to compare performance detriments and neurophysiologic evidence of sleep intrusion in an "ideal" sample of healthy controls and a "high-risk driver" sample of patients with excessive daytime sleepiness. 


\section{METHODS}

Recruitment and testing of healthy controls (Normals) and "high-risk driver" clinical patients with reports of excessive daytime sleepiness (EDS patients) was performed in compliance with University Health Network Research Ethics Board guidelines. Thirty healthy adults (mean age of $31.3 \pm 11.5)$ and 27 EDS patients $(47.0 \pm 13.7)$ with valid driver's licenses were included; normative subjects were screened for significant medical, psychiatric or sleep disorders, and use of psychotropic medications. EDS patients qualified by reporting an Epworth Sleepiness score $>10$ or reporting recent history of falling asleep while driving.

All subjects were read a standardized list of instructions relating to the driving performance task; they then were instructed to undertake a 15-minute driving test in the simulator to become familiarized with the performance task and to control for possible learning effects. Once familiarized with the task, subjects were tested for four 30-minute sessions at two hour intervals, occurring at 10:00, 12:00, 14:00 and 16:00h. Prior to each driving session, subjects were asked to complete instantaneous subjective ratings of sleepiness and alertness, using $10 \mathrm{~cm}$ lateral visual analogue scales (VAS). 3-channel polygraphic EEG/PSG data was collected simultaneous to digital collection of driving performance. Following completion of the final driving session, subjects were asked to provide brief qualitative feedback comments on their experience participating in the experiment.

Subjects were monitored closely by a research assistant during simulation drives, with the intent to maintain homogeneous testing conditions for all drives and avoid extraneous distractions or interruptions that could disturb the integrity of the experiment. Subjects were not allowed to nap or consume caffeinated beverages between sessions, and were asked to remain on-site between 30-minute testing sessions, but were permitted to be involved in any low-key activity they wished (e.g., reading, watching TV, etc.) within the hospital. The York Driving Simulator was used to assess driving performance. The driving simulator consists of a personal computer, 15" monitor and peripheral steering wheel, accelerator and brake accessories, and actual car seat. The simulator presents a forward view from the driver's seat of a motorway road scene, with standard lane markings and sign signals appropriate to the road environment. The specific driving environment used for this experiment was a monotonous two-lane highway with few turns, no stops signs or traffic lights, and posted speeds ranging from 70 to $100 \mathrm{~km} / \mathrm{h}$. Simulated wind gusts occurred at regular intervals, but were randomized with respect to direction and force, requiring the driver to periodically engage in compensatory corrective steering maneuvers to remain on the road, rather than holding the steering wheel in a rote "dead-man" position.

\section{Outcome Measures}

Driving performance was assessed using the following variables all expressed as a mean value over the 30-minute drive (continuous variables collected digitally@10 Hz):

1) Road position (tracking), measured as the deviation in percentile of the centre of the vehicle from the centre of the right hand lane. The extreme edge of the left lane position was demarcated as $100 \%$, and extreme right lane position was $0 \%$. Thus, ideal road position for the given task of maintaining the vehicle in the centre of the right lane was $25 \%$.

2) Speed. 
3) Speed deviation, calculated as the mean sum of differences in $\mathrm{km} / \mathrm{h}$ of the speed of the vehicle from the posted speed limits.

4) Crashes, calculated as the number of times that the simulated vehicle had off-road incidents, defined by the centre of the vehicle crossing over the extreme edge of the lane, or by blocking a passing vehicle in the left lane.

5) Steering reaction time (RT) to "virtual" wind gusts generated from either the left or right at random time intervals.

Neurophysiologic Monitoring. The number and duration of micro-sleep episodes were recorded by EEG during driving simulation sessions was the primary physiological dependent variable. Occipital (O2-A1) EEG lead was used as primary scoring lead, with bilateral submental EMG and right supraorbital EOG-01) leads used to corroborate evidence of sleep intrusion. Post-hoc scoring of episodes was performed blind to grouping of subject (Normal or EDS group) and Driving Performance data.

Total MS episodes for each ambulatory monitoring session included:

(a) minor MS - lapses of distinct sleep-related alpha activity or theta activity lasting 3 to 15 seconds;

(b) major MS - lapses greater than 15 seconds but less than 30 seconds, with EEG/PSG recording suggestive of any sleep stage.

Measured Subjective Scales. Immediately preceding each simulated drive, subjects were asked to complete instantaneous ratings of sleepiness and alertness, using $100 \mathrm{~mm}$ Visual Analogue Scales (VAS) of sleepiness (VAS-S) and alertness (VAS-A), scores expressed as 0-10.

\section{Statistical Analysis}

Independent t-tests were used to compare group differences between Normals and EDS Patients on all parameters. Mann-Whitney $U$ tests were used in place of t-tests in instances where the data distribution was significantly skewed. To analyze time-of-day effects, a repeated measure analysis was performed. Mean scores on the primary dependent variables were measured for each individual 30-minute testing period, and a composite mean score was also calculated using the mean of the four testing sessions. All data were analyzed and stored using the SPSS 11.5 program.

\section{RESULTS}

The following results were obtained for performance, physiological and subjective rating parameters described above. 
Table 1. Mean Speed $(\mathrm{km} / \mathrm{h})$

\begin{tabular}{|l|c|c|c|}
\hline Time & Norms & EDS & P \\
\hline $10: 00$ & $87.5+/-4.7$ & $88.5+/-4.9$ & .465 \\
\hline & & & \\
\hline $12: 00$ & $89.5+/-8.0$ & $89.2+/-6.2$ & .850 \\
\hline & & & \\
\hline $14: 00$ & $89.5+/-4.4$ & $89.1+/-4.7$ & .725 \\
\hline & & & \\
\hline $16: 00$ & $90.2+/-4.6$ & $90.8+/-7.6$ & .683 \\
\hline & & & \\
\hline Mean & $89.2+/-4.5$ & $89.4+/-5.3$ & .878 \\
\hline
\end{tabular}

$*$ signifies $\mathrm{p}<.05$

Table 3. Mean Road Position (\%)

\begin{tabular}{|l|c|c|c|}
\hline Time & Norms & EDS & $\mathrm{p}$ \\
\hline $10: 00$ & $29.8+/-5.4$ & $29.4+/-3.0$ & .713 \\
\hline & & & \\
\hline $12: 00$ & $28.3+/-3.9$ & $28.8+/-2.6$ & .609 \\
\hline & & & \\
\hline $14: 00$ & $28.5+/-3.9$ & $29.6+/-4.3$ & .293 \\
\hline & & & \\
\hline $16: 00$ & $28.9+/-4.3$ & $29.6+/-4.4$ & .562 \\
\hline & & & \\
\hline Mean & $28.9+/-4.1$ & $29.4+/-3.3$ & .635 \\
\hline
\end{tabular}

* signifies $\mathrm{p}<.05$

Table 5. Mean Crashes per Drive

\begin{tabular}{|c|c|c|c|}
\hline Time & Norms & EDS & $\mathrm{p}$ \\
\hline $10: 00$ & $1.2+/-1.7$ & $2.3+/-3.2$ & .098 \\
\hline & & & \\
\hline $12: 00$ & $1.3+/-1.7$ & $3.9+/-5.8$ & $\mathbf{. 0 3 1 *}$ \\
\hline & & & \\
\hline $14: 00$ & $1.6+/-2.0$ & $2.9+/-4.6$ & .168 \\
\hline & & & \\
\hline $16: 00$ & $1.3+/-1.3$ & $4.1+/-6.2$ & $\mathbf{. 0 3 4} *$ \\
\hline & & & \\
\hline Mean & $1.3+/-1.2$ & $3.2+/-4.2$ & $\mathbf{. 0 3 0 *}$ \\
\hline
\end{tabular}

$*$ signifies $\mathrm{p}<.05$
Table 2. Deviation from Speed Limit $(\mathrm{km} / \mathrm{h})$

\begin{tabular}{|c|c|c|c|}
\hline Time & Norms & EDS & $\mathrm{p}$ \\
\hline $10: 00$ & $-1.0+/-4.6$ & $-1.0+/-4.9$ & .979 \\
\hline & & & \\
\hline $12: 00$ & $1.2+/-7.9$ & $0.5+/-6.0$ & .717 \\
\hline & & & \\
\hline $14: 00$ & $1.1+/-4.2$ & $0.6+/-4.6$ & .666 \\
\hline & & & \\
\hline $16: 00$ & $1.6+/-4.4$ & $2.3+/-7.2$ & .632 \\
\hline & & & \\
\hline Mean & $.7+/-4.3$ & $.5+/-5.2$ & .840 \\
\hline \multicolumn{2}{|c|}{ * signifies $\mathrm{p}<.05$} &
\end{tabular}

Table 4. Mean Reaction Time (secs)

\begin{tabular}{|c|c|c|c|}
\hline Time & Norms & EDS & $\mathrm{p}$ \\
\hline $10: 00$ & $0.96+/-.45$ & $1.38+/-.32$ & $<.001 *$ \\
\hline & & & \\
\hline $12: 00$ & $1.05+/-.42$ & $1.55+/-.39$ & $<.001 *$ \\
\hline & & & \\
\hline $14: 00$ & $1.09+/-.39$ & $1.55+/-.48$ & $<.001 *$ \\
\hline & & & \\
\hline $16: 00$ & $1.04+/-.39$ & $1.54+/-.43$ & $<.001 *$ \\
\hline & & & \\
\hline Mean & $1.04+/-.39$ & $1.50+/-.37$ & $<.001 *$ \\
\hline
\end{tabular}

$*$ signifies $\mathrm{p}<.05$

Table 6. Microsleep Episodes per Drive

\begin{tabular}{|c|c|c|c|}
\hline Time & Norms & EDS & p \\
\hline $10: 00$ & $0.6+/-1.1$ & $3.6+/-5.1$ & $\mathbf{. 0 1 0 *}$ \\
\hline & & & \\
\hline $12: 00$ & $0.6+/-1.2$ & $2.7+/-3.3$ & $\mathbf{. 0 0 8 *}$ \\
\hline & & & \\
\hline $14: 00$ & $0.9+/-1.8$ & $2.9+/-3.7$ & $\mathbf{. 0 1 8 *}$ \\
\hline & & & \\
\hline $16: 00$ & $1.2+/-1.6$ & $3.0+/-3.5$ & $\mathbf{. 0 3 9 *}$ \\
\hline & & & \\
\hline Mean & $0.8+/-1.2$ & $3.6+/-4.3$ & $\mathbf{. 0 0 6 *}$ \\
\hline
\end{tabular}

$*$ signifies $\mathrm{p}<.05$ 
Table 7. Subjective Sleepiness (Visual Analogue Scale)

\begin{tabular}{|c|c|c|c|}
\hline Time & Norms & EDS & $\mathrm{p}$ \\
\hline $10: 00$ & $2.7+/-1.7$ & $4.4+/-2.1$ & $\mathbf{. 0 0 1} *$ \\
\hline & & & \\
\hline $12: 00$ & $2.2+/-1.7$ & $4.8+/-2.6$ & $<.001 *$ \\
\hline & & & \\
\hline $14: 00$ & $2.5+/-1.8$ & $6.1+/-2.4$ & $<.001 *$ \\
\hline & & & \\
\hline $16: 00$ & $2.6+/-2.1$ & $6.3+/-2.8$ & $<.001 *$ \\
\hline & & & \\
\hline Mean & $2.6+/-1.4$ & $5.4+/-1.8$ & $<.001 *$ \\
\hline
\end{tabular}

* signifies $\mathrm{p}<.05$
Table 8. Subjective Alertness (Visual Analogue Scale)

\begin{tabular}{|c|c|c|c|}
\hline Time & Norms & EDS & P \\
\hline $10: 00$ & $7.3+/-1.3$ & $5.6+/-2.4$ & $\mathbf{. 0 0 2} *$ \\
\hline & & & \\
\hline $12: 00$ & $7.4+/-1.8$ & $5.1+/-2.6$ & $<.00{ }^{*}$ \\
\hline & & & \\
\hline $14: 00$ & $7.6+/-1.6$ & $4.3+/-2.6$ & $<.00{ }^{*}$ \\
\hline & & & \\
\hline $16: 00$ & $7.2+/-2.1$ & $4.9+/-2.9$ & $\mathbf{. 0 0 2} *$ \\
\hline & & & \\
\hline Mean & $7.4+/-1.5$ & $5.0+/-2.0$ & $<.00{ }^{*}$ \\
\hline
\end{tabular}

* signifies $\mathrm{p}<.05$

\section{DISCUSSION}

We were able to demonstrate between-group differences using three separate approaches of measuring potential driving impairment due to sleepiness and reduced alertness.

"High-risk driver" patients with EDS did assess themselves as having higher levels of sleepiness and lower levels of alertness than healthy controls. Interestingly, diurnal variation was noted only for the parameter of sleepiness, but not for alertness (statistical comparison of circadian variation between groups not shown in this paper). Furthermore, only EDS patients showed a significant circadian fluctuation on self-ratings of sleepiness. Unlike previous research suggesting that drivers are generally able to assess their physiological level of sleepiness (Horne \& Baulk, 2004), our findings suggest that metacognition of fluctuations in consciousness may be poor for low levels of sleepiness, and individuals may not be aware of possible sleep-related impairments except at higher, and consequently, more dangerous levels of sleepiness. This would fit with a homeostatic model of metacognition relating to driver sleepiness, in which awareness of sleepiness appears as compensatory mechanisms overriding the physiological sleep drive increase. As these mechanisms begin to fail, conscious appreciation of sleepiness appears as a "last reserve" warning mechanism.

On driving simulator testing, the most sensitive measure demonstrating impairment in the EDS group compared to the normatives was RT. RT was fastest on the first drive of the day in both groups compared to subsequent drives, suggesting either a circadian fluctuation in psychomotor performance, task fatigue with a rote monotonous task, or a combination thereof. Mean speed and tracking did not differ between groups, although mean speed increased on repeated drives, which would appear concerning in light of elevated RT on afternoon drives. As vehicle crashes were relatively rare events, the statistical significance is harder to demonstrate with a modest sample size. However, using non-parametric tests clear overall elevations in crash incidence could be demonstrated. Furthermore, for EDS patients, there was a significant time-of-day effect between the first and last drives of the day, while for normals, no clear diurnal variation in crash incidence was seen. Thus it could be argued that although RT demonstrates definite trends in impairment-related crash risk, the low base rate of crashes may make this a less statistically robust simulator variable to use. 
While several other studies have demonstrated impairments in tracking ability, (i.e. standard deviation of road position) in sleep-disordered patients (Arnedt, Geddes \& MacLean 2005; Akerstedt et al., 2005; Contardi et al., 2004), the nature of this specific simulator task used here likely made this effect more difficult to detect. As the secondary performance task consisted of periodic countersteering maneuvers to simulated wind gusts, these made more subtle lane position deviations (analogous to "weaving") inherent to driving less statistically detectable in overall tracking comparisons.

On neurophysiologic testing, we have shown elevated levels of sleep intrusion for EDS patients at all four testing periods. Interestingly, on within-group comparisons for circadian effects, normals demonstrated a greater propensity towards MS on the final drive of the day compared to morning and noon-time; a similar phenomenon could not be demonstrated for the EDS group. Again, the relatively low overall base rate of MS events makes within-group comparisons difficult without an even larger sample size. Based on these and previous (Moller et al., 2002) observations, we would suggest that using the measure of brief MS events may be more sensitive than traditional daytime testing protocols such as MSLT/MWT to capture potentially dangerous fluctuations in cognitive capacities relevant to driving performance. Furthermore, this type of monitoring could be integrated into an in-vehicle scenario. Rather than using discrete events such as episodes of actual sleep or MS events, future research using a continuous spectral EEG analysis of theta and delta bands while driving might be an even more sensitive measure of sleep-proneness (Lal \& Craig, 2005).

It remains unclear whether the most accurate and clinically relevant measure of driving impairment due to sleepiness is derived from a neurophysiologic or performance-based approach. In a hospital- or clinic-based setting, combined simulator and EEG testing would appear a potentially valid tool to differentiate impairment due to intrinsic sleep pathologies. On road-side testing, these findings would support the potential use of standardized tests of reaction time, although these would likely not differentiate cause of impairment, and would address "state" rather than "trait" effects. We anticipate that forensic-industrial ("black-box") in-vehicle applications in the transport industry might benefit from multiple layers of information for greatest sensitivity and accuracy. User acceptance in the private transport sector will likely only increase with legislative changes.

\section{ACKNOWLEDGMENTS}

The first author is a student in the Collaborative Graduate Program of Institute of Medical Science and Knowledge Media Design at University of Toronto. Research funded through Postdoctoral Fellowship Awards by the Canadian Institute of Health Research (Institute of Neuroscience Mental Health and Addiction and Rx \& D Programs).

\section{REFERENCES}

Akerstedt, T., Peters, B., Anund, A., Kecklund, G. (2005). Impaired alertness and performance driving home from the night shift: a driving simulator study. Sleep Research, 14: 17-20.

Alchanatis, M., Zias, N., Deligiorgis, N., Amfilochiou, A., Dionellis, G., Orphanidou, D. (2005). Sleep apnea-related cognitive deficits and intelligence: an implication of cognitive reserve theory. Journal of Sleep Research 14: 69-75. 
Arnedt, J.T., Wilde, G.J., Munt, P.W., MacLean, A.W. (2000). Simulated driving performance following prolonged wakefulness and alcohol consumption: separate and contributions to impairment. Journal of Sleep Research, 9: 233-241.

Arnedt, J.T., Geddes, M.A.C., \& MacLean, A.W. (2005). Comparative sensitivity of a simulated driving task to subjective, physiological, and other performance measures during prolonged wakefulness. Journal of Psychosomatic Research, 58: 61-71.

Contardi, S., Pizza, F., Sancisi, E., Mondini, S., Cirignotta, F. (2004). Reliability of a driving simulation task for evaluation of sleepiness. Brain Research Bulletin 63: 427-31.

Determining Medical Fitness to Drive: A Guide for Physicians ( $6^{\text {th }}$ ed) (2000). Canadian Medical Association.

Horne, J.A. \& Baulk, S.D. (2004). Awareness of sleepiness when driving. Psychophysiology 41: 161-165.

Lal, S.K. \& Craig A. (2005). Reproducibility of the spectral components of the electroencephalogram during driver fatigue. International Journal of Psychophysiology, $55: 137-43$.

MacLean, A.W., Davies, D.R., Thiele, K. (2003). The hazards and prevention of driving while sleepy. Sleep Medicine Reviews, 7: 507-21.

Mitler, M., Carskaddon, M., Hirschkowitz, M. (2000). Daytime Tests. In, Principles and Practice of Sleep Medicine, $3^{\text {rd }}$ Ed. Kryger, M.H., Roth, T., Dement, W.C. (Eds). Saunders, 1251-8.

Moller, H., Kayumov, L. Shapiro, C.M. (2003). Microsleep Episodes, Attention Lapses and Circadian Variation in Psychomotor Performance in a Driving Simulation Paradigm. Proceedings of the $2^{\text {nd }}$ International Conference on Driving Assessment, 130-137.

Moller, H., Kayumov, L., Hossain, N., Shapiro C.M. (2002). Using a driving simulator to test alertness in patients presenting with excessive daytime sleepiness. Sleep, 25A:272.

Moller, H., Shapiro, C., Kayumov, L. (2004). Effects of Psychotropic Medications on Driving. In, Sleep and Sleep Disorders: Neuropsychopharmacological approach. Lader, M., Cardinali, D.P. \& Pandi-Perumal, S. (Eds). Landes Biosciences, 121-125.

Room, R., Babor, T. \& Rehm, J. (2005). Alcohol and public health. Lancet (365):519-30. 